Introduction Helpcenter (HC) is a low threshold centre for HIV and STI testing and dedicated to high risk groups: men having sex with men (MSM) and migrants from high endemic regions. Rapid HIV tests (RT) are used at HC for HIV screening since June 2007. The aim of this work is to describe our experience with this testing strategy.

Methods retrospective analyse of all RT routinely performed at HC. Tests are performed on plasma using the Determine Combo ${ }^{\oplus} \mathrm{HIV}-1 / 2 \quad \mathrm{Ag} / \mathrm{Ab}$ (Alere) test (4th generation). Determine ${ }^{\circledR} \mathrm{HIV}-1 / 2$ (3th generation) has been used until May 2009. All reactive tests were confirmed using INNO-LIA HIVI/II Score ${ }^{\circledR}$. A validation study was performed on 310 samples at the beginning of the project

Results up to December 2012, 5053 RT have been performed in 3884 persons including 1207 MSM and 994 migrants. The mean age was 33 years. 79 tests were reactive, 4968 negative and six not interpretable, two at the beginning of the project (learning curve) and four due to inappropriate light in the testing room and uncontrolled room temperature. Nine reactive tests were not confirmed with antibody or antigen tests (false-positive rate: $11.3 \%$ ). No false-negative results have been observed during the validation study. The prevalence of HIV was high among the risk groups: $2.9 \%$ of MSM and $3 \%$ of migrants tested were found hiv-positive. The availability of RT was an important reason to consult at HC, $66 \%$ of the respondents highlight it as motivation to consult at HC. RT is offered to each person consulting at $\mathrm{HC}, 90 \%$ of them accepted to undergo a RT as screening for HIV.

Conclusion the RT is well accepted in a dedicated testing centre. The overall prevalence of HIV infection was $1.8 \%$, nearly double in the target groups. Data on linkage to care will be presented.

\section{P5.025 DEVELOPMENT AND COMPARATIVE EVALUATION OF AN INNOVATIVE HIV SELF-TESTING SMARTPHONE APPLICATION, AN INTERNET-BASED AND A PAPER-BASED INSTRUCTIONAL PROGRAMME IN SOUTH AFRICA}

doi:10.1136/sextrans-2013-051184.1070

${ }^{1} \mathbf{N}$ Pai, 'T Behlim, ${ }^{1} \mathrm{R}$ Deli-Houssein, ${ }^{1} \mathrm{C}$ Vadnais, ${ }^{2} \mathrm{~L}$ Abrahams, ${ }^{2} \mathrm{~A}$ Binder, ${ }^{2} \mathrm{~K}$ Dheda. 'Mc Gill University, Montreal, OC, Canada; '2University of Cape Town, Cape Town, South Africa

Background South Africa has about 11\% of the total population living with HIV, the largest to date for any country. Facility-based HIV testing has reached only $50 \%$ South Africans because of fear of visibility leading to stigma, embarrassment and discrimination. Alternative strategies like self-testing for HIV may improve engagement, but evidence is limited. For self-testing to be successful, knowledge regarding the process, clear instructions about how to conduct, interpret and seek linkages to counselling and staging is essential.

Methods We created an internet-based HIV self-testing programme with a popular oral HIV test. The programme had built-in content for counselling, personal risk staging, instructions to selftest, and to seek counselling and referral. We also created an equivalent paper version and evaluated both programmes in 251 health care professionals working at University of Cape Town, South Africa. The tested internet programme was converted into an interactive, engaging smartphone HIV self-test application. The application was piloted for design, content and comprehension in 12 young adults (aged $18-25$ years).

Results Internet and paper-based self-testing programmes were well received $(91.3 \%)$ by participants with overall preference for self-testing reported at $100 \%$. User feedback on the smartphone application was incorporated after pilot evaluation and the following were improved: (a) a user centred design and layout, (b) colourful interface with clear instructions, (c) clarity of content for comprehension, (d) built-in features for expanded access, and (e) overall presentation. After six iterations, a prototype Android application was developed.

Conclusion High preference to self-test facilitated the use of the internet and paper-based programmes. This indicates that if validated self-tests are presented with clear instructions to self-test and built-in confidential linkages to counselling and treatment are provided, many more individuals will opt for HIV self-testing. These programmes and the smartphone application will be useful for the scale-up of unsupervised self-testing initiatives in literate populations worldwide.

\section{P5.026 MORE THAN JUST SELLING THE TEST: PHARMACIST OPINION ABOUT THE SALE OF OVER THE COUNTER HIV RAPID TESTS}

doi:10.1136/sextrans-2013-051184.1071

'B E Meyerson, ${ }^{2}$ P T Ryder, ${ }^{1} \mathrm{C}$ von Hippel, ${ }^{2} \mathrm{~K}$ Coy. ${ }^{1}$ Indiana University School of Public Health-Bloomington, Bloomington, IN, United States; ${ }^{2}$ Butler University College of Pharmacy and Health Sciences, Indianapolis, IN, United States

Community pharmacy sale of over-the-counter (OTC) rapid HIV tests may provide an important extension of the public health system. U.S. pharmacy practise has expanded to include several public health services; however, nothing is known about pharmacist attitudes about OTC sale of HIV tests and related consultation opportunities.

Methods This study identifies and explores the views of pharmacists regarding the sale of OTC rapid HIV tests. Exploratory interviews were conducted among a sample of 17 licenced community pharmacists in a Midwestern U.S. state with moderate HIV incidence. 30-minute interviews were conducted in person or by telephone between May and September 2012. Interviews were recorded and transcribed for a priori and open coding. Three investigators independently coded transcripts to assure interrator reliability.

Findings Pharmacists had positive attitudes about the OTC rapid HIV test, as testing would likely result in more people learning their HIV status. Participants felt that the pharmacy role should not be limited to test kit sale. Pharmacists framed their role as health consultants focused specifically on results consultation and linkage to treatment. Point of sale was identified as the opportunity for consultation about the HIV test and to establish a relationship for future discussion about results and linkage to care.

Conclusion Pharmacist consultation at point of OTC HIV test sale provides an important opportunity to increase options for linkage to HIV care. Future studies should investigate pharmacist opinion and attitudes about the OTC sale of rapid HIV test kits among a larger and more representative sample of community pharmacists, and in states with other geographic, socio demographic and epidemiologic characteristics.

\section{P5.027 HIGH PREVALENCE OF UNDIAGNOSED HIV INFECTION IN PATIENTS WHO WERE NOT OFFERED SCREENING AND PATIENTS WHO DECLINED SCREENING: EVALUATION OF A RAPID HIV SCREENING PROGRAMIME IN A U.S. URBAN EMERGENCY DEPARTMENT}

doi:10.1136/sextrans-2013-051184.1072

Y Hsieh, K Beck, G Kelen, J Shahan, C Kraus, 0 Laeyendecker, T Quinn, R Rothman. Johns Hopkins University, Baltimore, MD, United States

Background U.S. emergency departments(EDs) have become a cornerstone of the current CDC screening approach for identifying unrecognised HIV-infected patients. However, in spite of intensive efforts many ED screening programmes frequently fail to identify many infected patients. We aimed to investigate the prevalence of undiagnosed infection in an ED with an established HIV screening programme. 